\title{
A Worrying Decline in Anesthesia Journal Publications from Japan
}

\author{
Kazuyoshi Hirota
}

Received: 20 December 2012/Accepted: 26 December 2012/Published online: 23 January 2013

(C) Japanese Society of Anesthesiologists 2013

In Japan, a reduction in the supply of qualified physicians has become apparent since the introduction of a new postgraduate clinical training system in 2004. In addition, our younger generation seems to desire a medical specialist qualification but not the (research) degree of Doctor of Medicine. Indeed, in agreement with the results of a survey conducted by the Ministry of Health, Labour, and Welfare in $2005,92 \%$ of medical residents desired medical specialist qualifications whereas only $35 \%$ intended to obtain the degree of Doctor of Medicine (data from the home page of the Ministry of Health, Labour, and Welfare). As a consequence of high clinical workload and a relative lack of interest in research degrees, medical research paper submissions from Japan are decreasing [data from Web of Science (Thomson Reuters)]. Judging from the present state of publication numbers in major medical journals from our country, our world ranking in the major basic medical journals such as Nature Medicine, Cell, and Journal of Experimental Medicine remains high (3rd-4th position) whereas the ranking in major clinical medical journals such as New England Journal of Medicine, Lancet, and JAMA is declining (from 12th in 1993-1997 to 25th in 2008-2011). In addition, the ranking of South Africa, Brazil, India, Poland, Israel, Argentina, and Austria were previously (2003-2007) lower but now (2008-2011) higher than Japan. Overall numbers of clinical research papers from Japan have decreased by $8 \%$ whilst in the rest of the world they have increased by more than $10 \%$ [data from Web of Science (Thomson Reuters)].

K. Hirota $(\bowtie)$

Department of Anesthesiology, Hirosaki University Graduate

School of Medicine, Hirosaki 036-8562, Japan

e-mail: hirotak@cc.hirosaki-u.ac.jp
What about Anesthesiology publications from Japan? Li and colleagues [1] reported that publications from Japan have notably decreased in 17 anesthesiology journals including Anesthesiology, British Journal of Anaesthesia (BJA), Anesthesia and Analgesia (A\&A), Anaesthesia, Canadian Journal of Anesthesia, Acta Anaesthesiologica Scandinavica, European Journal of Anaesthesiology, Anaesthesia and Intensive Care, Journal of Neurosurgical Anesthesiology, Pediatric Anesthesia, International Journal of Obstetric Anesthesia, Journal of Clinical Anesthesia, Journal of Cardiothoracic and Vascular Anesthesia, Regional Anesthesia and Pain Medicine, Pain, European Journal of Pain, and Clinical Journal of Pain. In contrast, publications from China (including Hong Kong) and South Korea are increasing steadily. Using BJA Editor-inChief internal reports, I analyzed submission data to BJA for my presentation entitled, "Which Type of Articles from Japan Are Most Likely to Be Accepted by BJA?" in the BJA Session held at the 59th Annual Meeting of the Japanese Society of Anesthesiologists (JSA). I have found that the total number of submissions from Japan for the last 9 years placed 4 th in the world but there was a worrying trend that submission numbers declined year by year. As mentioned above, submissions from China and South Korea are increasing. Using PubMed, annual changes in publications in BJA showed a similar trend in these 3 East Asian countries. Annual publications in 2012 from Japan were one-third of those in 2003 (Fig. 1a). Annual publications in BJA from Japan were lower than those from South Korea over the last 4 years. I also analyzed publication data for Anesthesiology and A\&A for the last 10 years. Again, and of concern, I found that changes in annual publication numbers in Anesthesiology (Fig. 1b) and A\&A (Fig. 1c) were similar to those of BJA. In Anesthesiology, publications from Japan decreased 

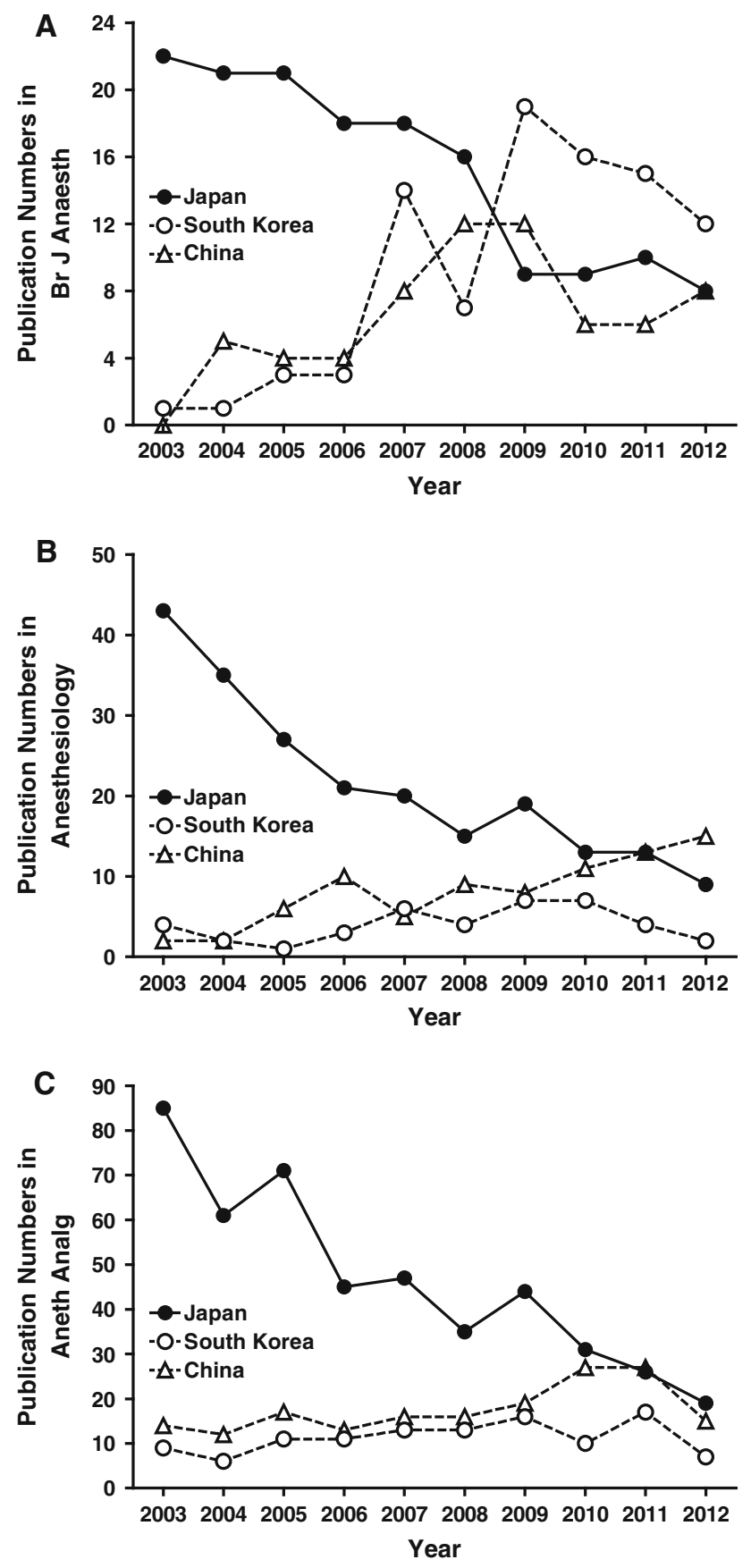

Fig. 1 Annual changes in publication numbers in (a) Br J Anaesth, (b) Anesthesiology, and (c) Anesth Analg

markedly whereas those from China increased, albeit modestly. Publications from Japan in 2012 were one-fifth of those in 2003, and were lower than those from China. Publications from Japan in A\&A also decreased substantially. Publications from Japan in 2012 were one-fourth of those in 2003.
Last year, the issue of data fabrication in randomized controlled clinical trials by Fujii had a major impact on the world of anesthesia [2]. I am extremely concerned that this may have a negative impact on the motivation within our specialty to perform research and consequently exacerbate the decrease in research publications that I have described. However, there are no such problems if clinical research is appropriately performed. Clinical investigations must be conducted according to the current Helsinki Declaration, be approved by research ethics committees, and proceed with written informed consent from all patients. In addition, before recruitment of the first patient, the clinical trial must be registered in a publicly accessible database such as the UMIN Clinical Trial Registry, Clinical Trials Information (JapicCTI), or Clinical Trial Registry in Japan. We, and in particular young anesthesiologists, should be concerned about the decrease in research publications from Japan compared to other East Asian countries. If our young generation loses its desire for research, Japan may play second fiddle to other East Asian countries in filed anesthesiology investigations. In a worst case anesthetic science may even become extinct in Japan. I agree with the following description in a 2008 BJA editorial [3]: "The key message is that anaesthetists should be scientists with an intellectual interest which extends beyond the walls of the operating room, the intensive care unit or the pain clinic. I would end as I began, with a provocative thought: a speciality without a research base and for anaesthesia this should include the basic sciences sits on the precipice of de-skilling and an almost inevitable plummet to into the service only abyss." If we, in Japan, do not engage in research activities, there is a real possibility that we may be viewed by our colleagues and patients simply as technicians. Our skillset may degenerate, and in the extreme case a medical degree may no longer be required. The way back from this situation, if there is one, would be difficult. Thus, I hope that, with strong mentorship and help from senior colleagues, young Japanese anesthesiologists will regain an appetite for research and help to put the brakes on this decline in research activity. The JSA should encourage and support this activity, and the time for action is NOW!

\section{References}

1. Li Z, Qiu LX, Wu FX, Yang LQ, Sun YM, Yu WF. Scientific publications in anesthesiology journals from East Asia: a 10-year survey of the literature. J Anesth. 2011;25:257-62.

2. Carlisle JB. The analysis of 168 randomised controlled trials to test data integrity. Anaesthesia. 2012;67:521-37.

3. Lambert DG. Volume 100: basic sciences in the British Journal of Anaesthesia. Br J Anaesth. 2008;100:595-6. 